\title{
Effect of Canagliflozin on Urinary Albumin Excretion in Japanese Patients with Type 2 Diabetes Mellitus and Microalbuminuria: A Pilot Study
}

\author{
Takeshi Osonoi, MD, PhD, Maki Gouda, BS, Mamiko Kubo, MSc, Kenji Arakawa, PhD, \\ Toshio Hashimoto, $\mathrm{BS}^{2}$, and Masanori Abe, $\mathrm{MD}, \mathrm{PhD}^{3}$
}

\begin{abstract}
Background: Albuminuria characterizes the progression of kidney injury. The effect of canagliflozin on the excretion of microalbumin was assessed for investigating its renoprotective potential in Japanese patients with type 2 diabetes mellitus (T2DM).

Patients and Methods: Twenty Japanese patients with T2DM and microalbuminuria were enrolled and administered with $100 \mathrm{mg}$ of canagliflozin once a day for 12 weeks. These subjects were admitted to the clinic at the start and end of the treatment period for 24-h urine collection. The primary endpoint was the percentage change in geometric mean 24-h urinary albumin excretion from baseline to week 12.

Results: The urinary albumin level decreased by $42.0 \%$ (95\% confidence interval: $21.9-57.0 ; P=0.0011)$ after 12 weeks of canagliflozin treatment. A number of blood and urinary parameters also significantly decreased, including hemoglobin A1c, fasting plasma glucose, estimated glomerular filtration rate, and creatinine clearance, while hematocrit was elevated. Among the biomarkers associated with kidney injury and inflammation, the urinary level of the oxidative stress marker 8-hydroxy-2'-deoxyguanosine was also decreased. There were no meaningful correlations noted between changes in urinary albumin excretion and other parameters/biomarkers. No severe adverse events were reported over the 12-week treatment period.

Conclusions: The results of this study indicate that canagliflozin decreases microalbuminuria in Japanese patients with T2DM. Albuminuria could be reduced as a result of changes in various physiological pathways; therefore, it is imperative that future, large-scale, studies attempt to determine the detailed mechanisms involved. Canagliflozin may offer a novel therapeutic option for Japanese patients with T2DM and incipient nephropathy.
\end{abstract}

Keywords: Canagliflozin, SGLT2 inhibitor, Type 2 diabetes, Albuminuria, Nephropathy, Renoprotective effect.

\section{Introduction}

D IABETIC NEPHROPATHY IS the most common cause of end-stage kidney disease (ESKD) and the incidence and prevalence of this condition have both dramatically in- creased worldwide. ${ }^{1,2}$ Both micro- and macroalbuminuria are predictors of progressive kidney diseases, as has been demonstrated in several prospective and epidemiological studies, particularly in patients with type 2 diabetes mellitus (T2DM). ${ }^{3,4}$ However, despite treatment with currently

\footnotetext{
${ }^{1}$ Department of Internal Medicine, Naka Kinen Clinic, Ibaraki, Japan.

${ }^{2}$ Ikuyaku, Integrated Value Development Division, Mitsubishi Tanabe Pharma Corporation, Tokyo, Japan.

${ }^{3}$ Division of Nephrology, Hypertension and Endocrinology, Department of Internal Medicine, Nihon University School of Medicine, Tokyo, Japan.

This study was presented at the 32nd Annual Meeting of the Japan Society of Diabetic Complications in October 2017 in Tokyo, Japan [Osonoi T, Abe M, Kubo M, et al.: Effect of canagliflozin on urinary albumin excretion in type 2 diabetic patients with microalbuminuria (pilot study).].

(C) Takeshi Osonoi, et al., 2018; Published by Mary Ann Liebert, Inc. This Open Access article is distributed under the terms of the Creative Commons License (http://creativecommons.org/licenses/by/4.0), which permits unrestricted use, distribution, and reproduction in any medium, provided the original work is properly credited.
} 
recommended therapy, numerous patients with T2DM exhibit significant albuminuria and progress toward ESKD. ${ }^{1,2}$ Previous studies have demonstrated a racial difference in the incidence of diabetic nephropathy and ESKD, and also that Asian patients with DM, as well as African Americans, Hispanics, and Native Americans, had a higher prevalence of diabetic nephropathy compared with Caucasian patients. ${ }^{5-9}$ In Japan, the percentage of new dialysis patients with diabetic nephropathy as the primary disease has been reported to be $43.8 \%$. $^{10}$ Therefore, an additional therapeutic strategy for preventing the progression of kidney disorders in patients with T2DM is urgently required, particularly for patients of specific ethnic groups, including those of Japanese origin.

Canagliflozin is a sodium glucose cotransporter 2 (SGLT2) inhibitor that increases the urinary excretion of glucose by inhibiting urinary glucose reabsorption in the proximal tubule. ${ }^{11}$ Several clinical studies have demonstrated that canagliflozin can control hyperglycemia and reduce body weight and blood pressure in patients with T2DM. ${ }^{12-15}$ In addition, SGLT2 inhibitors are thought to exert renoprotective effects by enhanced tubuloglomerular feedback, leading to a reduction in hyperfiltration, ${ }^{16-18}$ and by reducing inflammatory, fibrotic, and hyperplastic responses of proximal tubular cells via blocking glucose flow into the proximal tubule cells. ${ }^{19}$ In a phase 3 trial, compared with glimepiride, canagliflozin reduced urinary albumin excretion and caused an initial reduction in estimated glomerular filtration rate (eGFR), followed by a stabilization of kidney function. ${ }^{20}$ In the Canagliflozin Cardiovascular Assessment Study (CANVAS) Program, which aimed to assess cardiovascular outcomes, canagliflozin was also noted to suppress the progression of albuminuria and reduce composite renal outcomes in patients with T2DM with high cardiovascular risk. ${ }^{21}$ However, Japanese patients were not enrolled in these clinical studies. In phase 3 studies targeting Japanese patients with T2DM, albuminuria was only assessed in spot urine samples. ${ }^{12,13}$ This particular study showed slight reductions in albuminuria. However, because albumin levels in spot urine can be influenced by numerous factors, including circadian rhythm, hydration status, and physical activity, spot levels represent a less reliable measure for assessing renal impairment. ${ }^{22}$ Consequently, it was not concluded whether canagliflozin exerted renoprotective effects in these patients.

In the present study, we aimed to examine the renoprotective effect of canagliflozin in Japanese patients with T2DM and microalbuminuria via 24 -h urine collection. For further characterizing the effect of this treatment on kidney, we also investigated changes in biomarkers for kidney injury, inflammation, and oxidative stress.

\section{Patients and Methods}

Patients

Male and female patients (age 20-74 years) with T2DM exhibiting an albumin-to-creatinine ratio of $30.0-299.9 \mathrm{mg} / \mathrm{g}$ creatinine in a first-morning-void urinary test were selected. Subjects were treated using diet and exercise therapy alone, or in combination with antidiabetic drug therapy other than SGLT2 inhibitors, insulin, and glucagon-like peptide-1 receptor agonists. Other inclusion criteria were as follows: hemoglobin A1c $(\mathrm{HbA} 1 \mathrm{c}) \leq 10 \%$, systolic blood pressure
(SBP)/diastolic blood pressure (DBP) $<140 / 90 \mathrm{mmHg}$, and eGFR $\geq 45 \mathrm{~mL} / \mathrm{min} / 1.73 \mathrm{~m}^{2}$. eGFR was calculated according to the Japanese eGFR equation on the basis of serum creatinine (eGFRcreat). ${ }^{23}$

\section{Study design}

This study was a single-site, single-arm, open-label study aimed at evaluating the effect of canagliflozin on urinary microalbumin levels in patients with T2DM. We set our exploratory sample size at 20 subjects for feasibility of the study with an estimation accuracy of $95 \%$ confidence interval (CI), equating to $\sim 1 / 2$ of the standard deviation (SD). Canagliflozin $(100 \mathrm{mg})$ was administered once a day before or after breakfast for 12 weeks. Subjects were instructed not to change their diet and exercise regimen during the study and keep the dosage and administration of all concomitant medications constant. Subjects were admitted to the Naka Kinen Clinic 1 day before the start and end of the 12-week treatment period for 24-h urine collection. Spontaneous reports of adverse events (AEs) were collected from patients at the hospital visits. AEs were classified according to the Medical Dictionary for Regulatory Activities/Japanese Edition (MedDRA/J) version 20.0. This study was conducted between August 2016 and December 2017 in accordance with the Declaration of Helsinki (revised in October 2013). Written informed consent was obtained from all individuals before participation in this study. The protocol was registered in the UMIN Clinical Trial Registry as UMIN000023959 and was approved by the Institutional Ethics Review Committee of Naka Kinen Clinic. Members of the Clinical Operations Department, DOT World Co., Ltd. (Tokyo, Japan) performed monitoring and auditing in accordance with the Japanese governmental ethical guidelines and the study protocol.

\section{Endpoints}

The primary endpoint was the percentage change in geometric mean 24-h urinary albumin excretion from baseline to week 12 . Secondary endpoints included changes in laboratory data. Any AEs that occurred during the period of ongoing canagliflozin treatment were recorded.

The following parameters were measured in 24-h urine samples: albumin, transferrin, $N$-acetyl- $\beta$-D-glucosaminidase (NAG), liver-type fatty acid binding protein (L-FABP), $\beta 2$ microglobulin, 8-hydroxy-2'-deoxyguanosine (8-OHdG), aldosterone, and creatinine. The following urinary parameters were determined in first-morning-void urine samples, and the results expressed as a ratio to urinary creatinine as follows: albumin, type IV collagen, neutrophil gelatinaseassociated lipocalin (NGAL), kidney injury molecule-1 (KIM-1), and creatinine. Blood was collected for assessing blood biochemistry, including plasma interleukin (IL)-18, serum high-sensitivity C-reactive protein (CRP), plasma tumor necrosis factor receptor (TNFR) 1 and plasma TNFR2, serum erythropoietin, serum creatinine, serum cystatin $\mathrm{C}$, serum uric acid, plasma renin activity, and plasma aldosterone. All these parameters were measured at LSI Medience Corporation (Tokyo, Japan). HbA1c, fasting plasma glucose, body weight, blood pressure, 24-h water intake, and 24-h urine volume were measured at Naka Kinen Clinic. eGFR was calculated using both the Japanese eGFRcreat ${ }^{23}$ and the Japanese eGFR equation on the basis of serum cystatin $C$ 
(eGFRcys). ${ }^{24}$ Creatinine clearance was determined by serum creatinine and 24-h urinary creatinine excretion.

\section{Statistical analyses}

Data are presented as mean \pm SD or mean with $95 \%$ CI. Considering the skewed distribution of data, 24-h urinary albumin and the albumin-to-creatinine-ratio in first-morning-void urine samples were $\log$ transformed for analysis. The results of the mean changes and $95 \% \mathrm{CI}$ in the log transformed data from baseline to 12 weeks were back transformed (anti-log) to obtain geometric means of the ratios of the 12-week value to the baseline value and corresponding $95 \% \mathrm{CI}$. The values were then expressed as the percentage change from the respective baseline values. Changes from baseline were analyzed using two-tailed paired $t$-tests. Next, associations between the primary endpoint and other variables were assessed using Spearman's correlation coefficients. $P$-value of $<0.05$ was considered statistically significant. Because the statistical analyses of secondary endpoints were conducted exploratory, we did not consider multiplicity. All statistical analyses were performed with SAS software version 9.4 (SAS Institute, Inc., Cary, NC). Data management and statistical analyses were conducted by the Data Science Department, DOT World Co., Ltd.

\section{Results}

\section{Patients}

A total of 20 subjects ( 15 males and 5 females) were treated with $100 \mathrm{mg}$ of canagliflozin for 12 weeks. None of the subjects withdrew after consenting to the study. At baseline, these subjects had an HbA1c level of $7.30 \pm 0.91 \%$ and were $62.9 \pm 8.6$ years old. Mean body weight was $67.3 \pm 11.1 \mathrm{~kg}$, mean body mass index was $26.2 \pm 4.4 \mathrm{~kg} / \mathrm{m}^{2}$, and the mean duration of type 2 diabetes was $13.6 \pm 9.3$ years. Twelve patients $(60 \%)$ were taking an angiotensin receptor blocker.

\section{Primary endpoint}

As shown in Figure 1, patients had a geometric mean 24-h urinary albumin of $142.4 \mathrm{mg} / \mathrm{day}$ at baseline and $82.6 \mathrm{mg} / \mathrm{day}$ after 12 weeks of treatment with canagliflozin. The geometric mean 24-h urinary albumin significantly decreased by $42.0 \%$ $(P=0.0011$, Fig. 1). For reference, we also assessed the effect on albumin-to-creatinine ratio using first-morning-void urine samples. The geometric mean albumin-to-creatinine ratio tended to decrease by $22.1 \%(P=0.0876$; Table 1$)$.

\section{Fasting plasma glucose, body weight, HbA1c, and blood pressure}

Twelve weeks of treatment with $100 \mathrm{mg}$ canagliflozin significantly reduced fasting plasma glucose and HbAlc by $24 \mathrm{mg} / \mathrm{dL}$ and $0.24 \%$, respectively (Table 2 ). Body weight also significantly decreased by $1.7 \mathrm{~kg}$, whereas no significant differences were detected in SBP and DBP (Table 2).

\section{eGFR and creatinine clearance}

eGFRcreat and eGFRcys significantly decreased by $8.9 \mathrm{~mL} / \mathrm{min} / 1.73 \mathrm{~m}^{2}$ and $8.0 \mathrm{~mL} / \mathrm{min} / 1.73 \mathrm{~m}^{2}$, respectively (Table 3). Creatinine clearance also decreased by $16.9 \mathrm{~mL} /$ $\min / 1.73 \mathrm{~m}^{2}$ (Table 3).

\section{Biomarkers for tissue damage, oxidative stress, inflammation, and the renin-angiotensin-aldosterone system}

Next, we investigated a range of markers for glomerular damage. While the urinary transferrin level was significantly

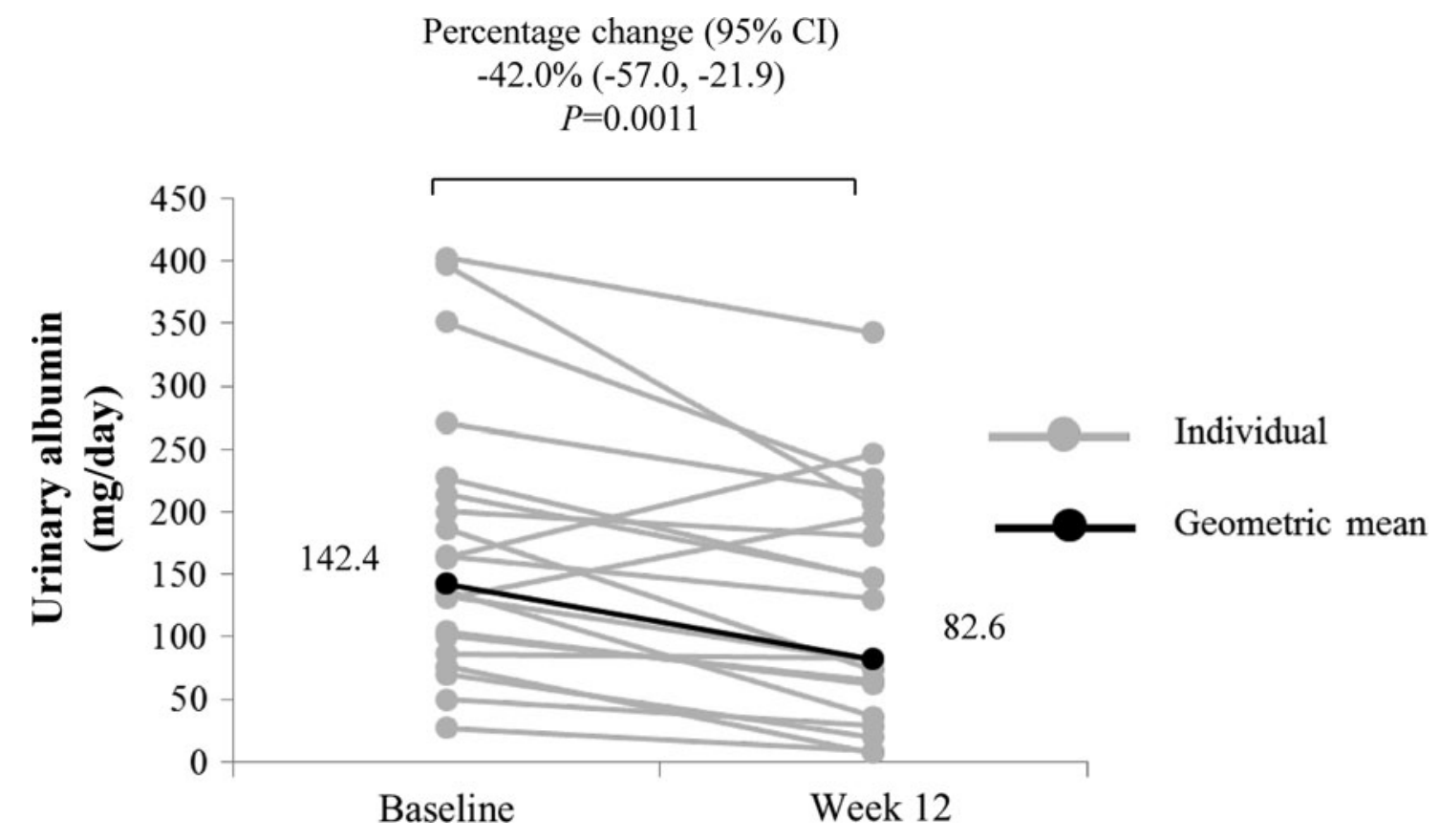

FIG. 1. Change in 24-h urinary albumin from baseline to week 12. Data are expressed as individual measurements (gray circles) and geometric mean (black circles and values in the figure). Statistical comparison for the change in geometric mean from baseline to week 12 was conducted using a paired $t$-test. Arithmetic mean \pm SD at baseline and week 12 were $174.7 \pm 109.6$ and $125.2 \pm 93.5 \mathrm{mg} / \mathrm{dL}$, respectively. SD, standard deviation. 
Table 1. Change in Albumin-to-Creatinine Ratio in First-Morning-Void Urine from Baseline to Week 12

\begin{tabular}{lcccc}
\hline Variable & Baseline & Week 12 & Percentage change (95\% CI) & $\mathrm{P}$ value \\
\hline Albumin-to-creatinine ratio, $\mathrm{mg} / \mathrm{g} \cdot \mathrm{Cr}$ & 86.1 & 67.1 & $-22.1(-41.7$ to 4.1$)$ & 0.0876 \\
\hline
\end{tabular}

Data are expressed as geometric mean $(95 \% \mathrm{CI})$ and $P$-value by paired $t$-test $(n=20)$. Arithmetic mean \pm SD in albumin-to-creatinine ratio at baseline and week 12 were $104.6 \pm 84.4$ and $105.0 \pm 99.8 \mathrm{mg} / \mathrm{g} \cdot \mathrm{Cr}$, respectively.

$\mathrm{CI}$, confidence interval; SD, standard deviation.

decreased after 12 weeks of treatment with canagliflozin (Table 4), the level of urinary type IV collagen did not change significantly. Regarding urinary markers for tubular injury, L-FABP and NGAL significantly decreased and increased, respectively, after 12 weeks of canagliflozin treatment (Table 4). Urinary NAG, $\beta 2$-microglobulin, and KIM-1 remained unchanged, while urinary $8-\mathrm{OHdG}$, a marker of oxidative stress, was significantly decreased after 12 weeks of treatment with canagliflozin. Levels of inflammatory markers, such as plasma IL-18 and serum high-sensitivity CRP, did not significantly change. Plasma TNFR2 levels significantly increased, while TNFR1 levels did not significantly change. Plasma aldosterone levels and renin activity were both significantly increased, while urinary aldosterone did not significantly change.

\section{Hematocrit, water intake, urinary volume,} serum uric acid, and erythropoietin

Hematocrit significantly increased by $3.0 \%$, whereas erythropoietin and serum uric acid levels did not significantly change (Table 5). Water intake and urinary volume significantly increased by 432 and $345 \mathrm{~mL} /$ day, respectively (Table 5). There was a positive correlation noted between changes in water intake and urinary volume.

\section{Correlation between changes in 24-h urinary albumin and other parameters}

The change in 24-h urinary albumin was significantly correlated with those in urinary transferrin and plasma renin activity $(r=0.651 ; P=0.0019$ and $r=-0.468 ; P=0.0374$, respectively, Table 6 ). There were no other significant correlations between the change in 24-h urinary albumin and changes in other parameters, including HbA1c, fasting plasma glucose, body weight, and eGFR.

\section{Safety}

Over the 12 weeks of canagliflozin treatment, there were no reports of serious AEs. There was one event involving upper respiratory infection in one subject and one event of muscle pain in another subject; both these patients recovered during the treatment period. In addition, there were no notable changes in laboratory test values associated with the safety of subjects.

\section{Discussion}

The development of albuminuria not only characterizes the progression of kidney injury ${ }^{3,4}$ but it can also predict cardiovascular risk in patients with T2DM. ${ }^{25}$ Therefore, the guidelines recommended the regular assessment of albuminuria and reducing albuminuria in patients with T2DM. ${ }^{26,27}$ However, many patients with T2DM worldwide, including Japan, have substantial residual albuminuria and continue to progress toward ESKD. Thus, additional treatment complementing the existing therapies remains an important unmet medical need. The present pilot study showed that 12 weeks of treatment with canagliflozin significantly reduced 24 -h urinary albumin excretion in Japanese patients with T2DM and microalbuminuria.

In the present study, canagliflozin reduced 24-h urinary albumin excretion by $42 \%$. A similar magnitude of reduction in albuminuria was previously reported following canagliflozin treatment in Western patients with T2DM. ${ }^{20}$ Furthermore, consistent with the results of previous non-Japanese studies, ${ }^{28-30}$ the changes in HbA1c, fasting plasma glucose, blood pressure, and body weight did not correlate with the albuminuria response during canagliflozin treatment. Thus, canagliflozin appears to reduce urinary albumin excretion in patients with T2DM without racial differences.

We also observed that the levels of urinary transferrin were reduced after 12 weeks of treatment with canagliflozin. Transferrin is a biomarker for glomerular damage. Furthermore, it is well known that urinary transferrin showed a high correlation with urinary albumin. ${ }^{31}$ Taken together, the amelioration of damaged glomerular function in these patients is suggested. In addition to glomerular damage, recent studies have also shown the involvement of tubular damage during the pathogenesis of diabetic nephropathy. ${ }^{32,33}$ However, the tubular damage markers evaluated in this study were all within

Table 2. Changes in Fasting Plasma Glucose, Body Weight, HiA1c, SBP, AND DBP FROM BASELINE TO WEEK 12

\begin{tabular}{|c|c|c|c|c|}
\hline Variables & Baseline & Week 12 & Change & $\mathrm{P}$ value \\
\hline Fasting plasma glucose, mg/dL & $151 \pm 22$ & $128 \pm 18$ & $-24 \pm 16(-31$ to -16$)$ & $<0.0001$ \\
\hline Body weight, kg & $67.3 \pm 11.1$ & $65.6 \pm 11.1$ & $-1.7 \pm 1.4(-2.4$ to -1.1$)$ & $<0.0001$ \\
\hline $\mathrm{HbA} 1 \mathrm{c}, \%$ & $7.30 \pm 0.91$ & $7.07 \pm 0.64$ & $-0.24 \pm 0.45(-0.45$ to -0.02$)$ & $\mathbf{0 . 0 3 1 8}$ \\
\hline $\mathrm{SBP}, \mathrm{mmHg}$ & $134.3 \pm 11.6$ & $131.9 \pm 8.9$ & $-2.4 \pm 15.6(-9.7$ to 4.9$)$ & 0.4998 \\
\hline $\mathrm{DBP}, \mathrm{mmHg}$ & $81.3 \pm 9.6$ & $80.4 \pm 9.9$ & $-0.9 \pm 9.5(-5.3$ to 3.6$)$ & 0.6925 \\
\hline
\end{tabular}

Data are expressed as mean $\pm \mathrm{SD}$, mean $\pm \mathrm{SD}(95 \% \mathrm{CI})$, and $P$-value by paired $t$-test $(n=20)$. Bold texts indicate $P$-value less than 0.05 . DBP, diastolic blood pressure; HbA1c, hemoglobin A1c; SBP, systolic blood pressure. 
Table 3. Changes in eGFR and Creatinine Clearance FROM BASELINE TO WEEK 12

\begin{tabular}{lrrrr}
\hline Variables & Baseline & Week 12 & Change & P value \\
\hline eGFRcreat, $\mathrm{mL} / \mathrm{min} / 1.73 \mathrm{~m}^{2}$ & $77.5 \pm 18.1$ & $68.6 \pm 15.2$ & $-8.9 \pm 7.5(-12.4$ to -5.3$)$ & $<\mathbf{0 . 0 0 0 1}$ \\
eGFRcys, $\mathrm{mL} / \mathrm{min} / 1.73 \mathrm{~m}^{2}$ & $83.1 \pm 22.8$ & $75.2 \pm 21.0$ & $-8.0 \pm 7.7(-11.6$ to -4.3$)$ & $\mathbf{0 . 0 0 0 2}$ \\
Creatinine clearance, $\mathrm{mL} / \mathrm{min} / 1.73 \mathrm{~m}^{2}$ & $107.5 \pm 26.3$ & $90.6 \pm 23.3$ & $-16.9 \pm 17.0(-24.8$ to -8.9$)$ & $\mathbf{0 . 0 0 0 3}$ \\
\hline
\end{tabular}

Data are expressed as mean $\pm \mathrm{SD}$, mean $\pm \mathrm{SD}(95 \% \mathrm{CI})$, and $P$-value by paired $t$-test $(n=20)$. Bold texts indicate $P$-value less than 0.05 . eGFRcreat, estimated glomerular filtration rate based on creatinine; eGFRcys, estimated glomerular filtration rate based on serum cystatin C.

the reference ranges both at baseline and at week 12. There was a small decrease in urinary L-FABP level, which reflected the mitigation of proximal tubular stress presumably via reducing the amount of work required following canagliflozin treatment. ${ }^{34-36}$ Even though this could increase albumin reabsorption, it is unlikely that increased tubular albumin reabsorption plays a major role in the reduced level of urinary albumin excretion.

In this study, eGFR was lower following 12 weeks of canagliflozin treatment. Previous investigations on SGLT2 inhibitors, including canagliflozin, putatively by activated tubuloglomerular feedback, ${ }^{37}$ showed an acute decline in eGFR followed by a period of maintained kidney function. ${ }^{20,29,38,39}$ A similar reduction in eGFR was reported with renin-angiotensin-aldosterone system inhibitors, ${ }^{40-42}$ and this would suggest that an intraglomerular hemodynamic effect, including the alleviation of glomerular hypertension, contributes to the reduction of albuminuria. ${ }^{43,44}$ In addition, the activation of inflammatory pathways and oxidative stress has been shown in the kidneys of patients with diabetic nephropathy, ${ }^{45,46}$ and is estimated to be a factor that initiates and sustains disease progression. However, the inflammatory biomarkers measured in this study were not suppressed and circulating TNFR2 levels slightly increased after canagliflozin treatment. Intriguingly, urinary levels of the oxidative stress marker $8-\mathrm{OHdG}$ were reduced after treatment with canagliflozin. Moreover, hematocrit was elevated after treatment with canagliflozin, which is expected to deliver more oxygen to tissues to reduce ischemic stress. ${ }^{34,35}$ However, we did not observe any association between changes in 24-h urinary albumin excretion and either of the abovementioned variables, including eGFR, 8-OHdG, and hematocrit. Although the change in plasma renin activity was significantly correlated with change in urinary albumin, we could not conclude that it was a real finding, because the correlation was driven almost exclusively by a single subject with a very large decrease in 24-h urinary albumin and no clear trend was observed in the rest of the subjects. As the present study cannot provide conclusive evidence for the precise mechanism involved, we must assume that multiple characteristics of the drug could be involved in reducing albuminuria.

There were two AEs, and no serious AEs, reported during the treatment period. In a previous study in patients with T2DM and stage 3 chronic kidney disease (eGFR: $30-59 \mathrm{~mL} / \mathrm{min} / 1.73 \mathrm{~m}^{2}$ ), genital mycotic infections and volume depletion-related AEs were frequently observed in the canagliflozin-treated group (number of patients/number of participants [event rate]: 51/703 [7.3\%] and 48/703 [6.8\%], respectively) than placebo group $(6 / 382[1.6 \%]$ and $10 / 382[2.6 \%]$, respectively $),{ }^{47}$ and some reports have warned that SGLT2 inhibitors could induce acute kidney injury. ${ }^{48,49}$ However, no volume depletion- or renal-related AEs

Table 4. Changes in Biomarkers of Tissue Damage, Oxidative Stress, Inflammation, and the Renin-Angiotensin-Aldosterone System from Baseline to Week 12

\begin{tabular}{|c|c|c|c|c|}
\hline Variables & Baseline & Week 12 & Change & $\mathrm{P}$ value \\
\hline Urinary transferrin, $\mathrm{mg} / 24 \mathrm{~h}$ & $13.8 \pm 8.9$ & $10.8 \pm 8.0$ & $-3.0 \pm 4.7(-5.2$ to -0.8$)$ & 0.0104 \\
\hline Urinary type IV collagen, $\mu \mathrm{g} / \mathrm{g} \cdot \mathrm{Cr}$ & $3.9 \pm 2.3$ & $3.9 \pm 1.6$ & $0.0 \pm 2.0(-1.0$ to 0.9$)$ & 0.9913 \\
\hline Urinary NAG, U/24h & $8.4 \pm 3.6$ & $8.5 \pm 3.1$ & $0.1 \pm 2.7(-1.2$ to 1.3$)$ & 0.9013 \\
\hline Urinary L-FABP, $\mu \mathrm{g} / 24 \mathrm{~h}$ & $5.3 \pm 7.2$ & $3.5 \pm 5.7$ & $-1.8 \pm 2.6(-3.0$ to -0.6$)$ & 0.0055 \\
\hline Urinary $\beta 2$ microglobulin, $\mu \mathrm{g} / 24 \mathrm{~h}$ & $145 \pm 229$ & $200 \pm 517$ & $55 \pm 322(-96$ to 205$)$ & 0.4586 \\
\hline Urinary NGAL, $\mathrm{ng} / \mathrm{mg} \cdot \mathrm{Cr}$ & $12.3 \pm 25.7$ & $18.9 \pm 37.7$ & $6.6 \pm 13.3(0.4-12.9)$ & 0.0381 \\
\hline Urinary $\mathrm{KIM}-1, \mathrm{ng} / \mathrm{mg} \cdot \mathrm{Cr}$ & $1.5 \pm 0.9$ & $1.4 \pm 0.7$ & $-0.1 \pm 0.7(-0.4$ to 0.2$)$ & 0.5806 \\
\hline Urinary $8-\mathrm{OHdG}, \mu \mathrm{g} / 24 \mathrm{~h}$ & $7.0 \pm 5.1$ & $4.6 \pm 3.9$ & $-2.4 \pm 4.3(-4.5$ to -0.4$)$ & 0.0212 \\
\hline Plasma IL-18, pg/mL & $313 \pm 111$ & $321 \pm 106$ & $8 \pm 94(-36$ to 52$)$ & 0.7051 \\
\hline Serum high-sensitivity CRP, mg/L & $0.9 \pm 1.3$ & $1.1 \pm 1.6$ & $0.2 \pm 1.4(-0.5$ to 0.9$)$ & 0.5148 \\
\hline Plasma TNFR1, pg/mL & $1256 \pm 217$ & $1220 \pm 186$ & $-35 \pm 143(-102$ to 31$)$ & 0.2815 \\
\hline Plasma TNFR2, pg/mL & $2587 \pm 600$ & $2826 \pm 605$ & $239 \pm 502(4-473)$ & 0.0468 \\
\hline Plasma aldosterone, $\mathrm{pg} / \mathrm{mL}$ & $94.8 \pm 39.2$ & $121.4 \pm 48.8$ & $26.6 \pm 43.4(6.3-46.9)$ & 0.0130 \\
\hline Plasma renin activity, $\mathrm{ng} /(\mathrm{mL} \cdot \mathrm{h})$ & $2.0 \pm 2.8$ & $4.5 \pm 5.8$ & $2.5 \pm 3.9(0.7-4.3)$ & 0.0092 \\
\hline Urinary aldosterone, $\mu \mathrm{g} / 24 \mathrm{~h}$ & $4.5 \pm 4.1$ & $4.7 \pm 3.0$ & $0.3 \pm 2.7(-1.0$ to 1.5$)$ & 0.6669 \\
\hline
\end{tabular}

Data are expressed as mean $\pm \mathrm{SD}$, mean $\pm \mathrm{SD}(95 \% \mathrm{CI})$, and $P$-value by paired $t$-test $(n=20)$. Bold texts indicate $P$-value less than 0.05 . CRP, C-reactive protein; 8-OHdG, 8-hydroxy-2'-deoxyguanosine; IL-18, interleukin-18; KIM-1, kidney injury molecule-1; L-FABP, liver-type fatty acid binding protein; NAG, $N$-acethyl- $\beta$-D-glucosaminidase; NGAL, neutrophil gelatinase-associated lipocalin; TNFR, tumor necrosis factor receptor. 
Table 5. Changes in Hematocrit, Water Intake, Urinary Volume, Serum Uric Acid, AND ERYTHROPOIETIN FROM BASELINE TO WEEK 12

\begin{tabular}{lcccr}
\hline Variables & Baseline & Week 12 & Change & P value \\
\hline Hematocrit, \% & $41.7 \pm 4.6$ & $44.7 \pm 4.7$ & $3.0 \pm 2.0(2.0-3.9)$ & $<\mathbf{0 . 0 0 0 1}$ \\
Water intake, mL/day & $2272 \pm 1145$ & $2704 \pm 1078$ & $432 \pm 693(108-756)$ & $\mathbf{0 . 0 1 1 8}$ \\
Urinary volume, mL/day & $2759 \pm 1019$ & $3105 \pm 1208$ & $345 \pm 686(24-666)$ & $\mathbf{0 . 0 3 6 4}$ \\
Serum uric acid, mg/dL & $5.6 \pm 1.4$ & $5.4 \pm 1.3$ & $-0.2 \pm 0.8(-0.6$ to 0.2$)$ & 0.2285 \\
Erythropoietin, mIU/mL & $9.8 \pm 5.3$ & $9.7 \pm 5.7$ & $-0.1 \pm 3.7(-1.8$ to 1.7$)$ & 0.9245 \\
\hline
\end{tabular}

Data are expressed as mean $\pm \mathrm{SD}$, mean $\pm \mathrm{SD}(95 \% \mathrm{CI})$, and $P$-value by paired $t$-test $(n=20)$. Bold texts indicate $P$-value less than 0.05 .

were reported in our present short-term study, and no novel safety concerns were raised.

There are some limitations to this study that should be taken into consideration. The study was performed in a single-site, single-arm, and open-label manner, sample size was relatively small, and the study follow-up period was only 12 weeks. The results generated by the present study, therefore, warrant randomized-controlled, multicenter trials with a sufficiently large number of participants and a longer treatment period for fully confirming the renoprotective effects and for delineating the mechanism of action. Definitive evidence of the renoprotective effects of canagliflozin is expected to be provided by the following ongoing studies: Canagliflozin and Renal Endpoints in Diabetes with Estab-

Table 6. Correlation Analysis Between Changes in 24-Hour Urinary Albumin and Other Variables

\begin{tabular}{lcc}
\hline & $\begin{array}{c}\text { Spearman's } \\
\text { correlation } \\
\text { coefficient }\end{array}$ & P values \\
Variables & 0.651 & $\mathbf{0 . 0 0 1 9}$ \\
Urinary transferrin & -0.468 & $\mathbf{0 . 0 3 7 4}$ \\
Plasma renin activity & 0.423 & 0.0628 \\
Urinary L-FABP & 0.422 & 0.0637 \\
Urinary NAG & 0.392 & 0.0875 \\
SBP & -0.331 & 0.1536 \\
Serum uric acid & -0.330 & 0.1551 \\
Plasma aldosterone & 0.326 & 0.1610 \\
HbA1c & 0.323 & 0.1644 \\
DBP & -0.315 & 0.1756 \\
eGFRcys & -0.290 & 0.2156 \\
Serum high-sensitivity CRP & -0.266 & 0.2577 \\
Urinary aldosterone & 0.263 & 0.2619 \\
Urinary $\beta 2$ microglobulin & -0.230 & 0.3291 \\
Plasma IL-18 & 0.217 & 0.3588 \\
Hematocrit & -0.184 & 0.4364 \\
Fasting plasma glucose & 0.161 & 0.4976 \\
Creatinine clearance & 0.134 & 0.5738 \\
Plasma TNFR2 & 0.126 & 0.5952 \\
Erythropoietin & -0.120 & 0.6134 \\
Water intake & 0.078 & 0.7431 \\
Urinary volume & 0.067 & 0.7782 \\
Body mass index & 0.065 & 0.7865 \\
Body weight & -0.041 & 0.8649 \\
Urinary type IV collagen & -0.041 & 0.8650 \\
Urinary NGAL & 0.032 & 0.8922 \\
eGFRcreat & 0.032 & 0.8923 \\
Plasma TNFR1 & -0.026 & 0.9148 \\
Urinary KIM-1 & -0.002 & 0.9950 \\
Urinary 8-OHdG & & \\
\hline & & \\
& &
\end{tabular}

$n=20$. Bold texts indicate $P$-value less than 0.05 . lished Nephropathy Clinical Evaluation Study (CREDENCE; ClinicalTrials.gov number, NCT02065791) and Effect of Canagliflozin in Type 2 Diabetic Patients with Microalbuminuria in Japanese Population (CANPIONE; UMIN trial ID: UMIN000029905).

\section{Conclusions}

The findings of this pilot study revealed that canagliflozin significantly decreases microalbuminuria after 12 weeks of treatment in Japanese patients with T2DM. The primary mechanism for the reduction of albuminuria was not elucidated but appears to be mediated by multiple mechanisms. Canagliflozin and other SGLT2 inhibitors may offer a novel therapeutic option for Japanese patients with T2DM and incipient nephropathy.

\section{Acknowledgments}

The authors thank Mr. Fujio Matsuyama (DOT World Co., Ltd.) for performing the statistical analysis, Mr. Toru $\mathrm{Ku}-$ magai (DOT World Co., Ltd.) for providing medical writing support funded by Mitsubishi Tanabe Pharma Corporation, and Makoto Ueno, PhD (Pharmacovigilance Department, Mitsubishi Tanabe Pharma Corporation), for useful advice. This study was funded by Mitsubishi Tanabe Pharma Corporation. Canagliflozin (Japanese brand name: Canaglu) was developed, manufactured, and distributed by Mitsubishi Tanabe Pharma Corporation.

\section{Authors' Contributions}

T.O. contributed to the study design and conducted the study as the principal investigator. M.A. was the medical advisor for this study and contributed to the study design. M.G., M.K., K.A., and T.H. contributed to the study design. All authors contributed to the interpretation of data, writing, and reviewing the article and approved this article for submission.

\section{Author Disclosure Statement}

T.O. received consulting fees and/or speaker bureaus from Astellas Pharma, Inc., Mitsubishi Tanabe Pharma Corporation, Novo Nordisk Pharma Ltd., Ono Pharmaceutical Co., Ltd., Sanwa Kagaku Kenkyusho Co., Ltd., and Takeda Pharmaceutical Co., Ltd., and received research support from Astellas Pharma, Inc., Abbott Japan Co., Ltd., AbbVie GK, Bayer Yakuhin, Ltd., Daiichi Sankyo Co., Ltd., Eli Lilly Japan K.K., Japan Tobacco, Inc., Kowa Company, Ltd., Mitsubishi Tanabe Pharma Corporation, Nippon Boehringer 
Ingelheim Co., Ltd., Novo Nordisk Pharma Ltd., Sanofi K.K., Sanwa Kagaku Kenkyusho Co., Ltd., Taisho Pharmaceutical Co., Ltd., and Takeda Pharmaceutical Co., Ltd.

M.A. received consulting fees and/or speaker bureaus from Eli Lilly Japan K.K., Kyowa Hakko Kirin Co., Ltd., Mitsubishi Tanabe Pharma Corporation, Otsuka Pharmaceutical Co., Ltd., and Nippon Boehringer Ingelheim Co., Ltd.; received scholarship grants from Astellas Pharma, Inc., Baxter Limited, Bayer Yakuhin, Ltd., Daiichi Sankyo Co., Ltd., Kyowa Hakko Kirin Co., Ltd., Mitsubishi Tanabe Pharma Corporation, NIPRO Corporation, Otsuka Pharmaceutical Co., Ltd., Pfizer Japan, Inc., Shionogi \& Co., Ltd., and Torii Pharmaceutical Co., Ltd.; and chair courses endowed by Chugai Pharmaceutical Co., Ltd., Nikkiso Co., Ltd., NIPRO Corporation, Ono Pharmaceutical Co., Ltd., Otsuka Pharmaceutical Co., Ltd., Terumo Corporation, and Toray Medical Co., Ltd.

M.G., M.K., K.A., and T.H. are employees of Mitsubishi Tanabe Pharma Corporation.

\section{References}

1. Shahbazian H, Rezaii I: Diabetic kidney disease; review of the current knowledge. J Renal Inj Prev 2013;2:73-80.

2. Tomino Y, Gohda T: The prevalence and management of diabetic nephropathy in Asia. Kidney Dis (Basel) 2015;1: 52-60.

3. Ninomiya T, Perkovic V, de Galan BE, et al.: Albuminuria and kidney function independently predict cardiovascular and renal outcomes in diabetes. J Am Soc Nephrol 2009;20: 1813-1821.

4. Katayama S, Moriya T, Tanaka S, et al.: Low transition rate from normo- and low microalbuminuria to proteinuria in Japanese type 2 diabetic individuals: the Japan Diabetes Complications Study (JDCS). Diabetologia 2011;54:10251031.

5. Cowie CC, Port FK, Wolfe RA, et al.: Disparities in incidence of diabetic end-stage renal disease according to race and type of diabetes. N Engl J Med 1989;321:1074-1079.

6. Stephens GW, Gillaspy JA, Clyne D, et al.: Racial differences in the incidence of end-stage renal disease in types I and II diabetes mellitus. Am J Kidney Dis 1990;15:562567.

7. Chandie Shaw PK, Baboe F, van Es LA, et al.: South-Asian type 2 diabetic patients have higher incidence and faster progression of renal disease compared with DutchEuropean diabetic patients. Diabetes Care 2006;29:13831385.

8. Parving HH, Lewis JB, Ravid M, et al.: Prevalence and risk factors for microalbuminuria in a referred cohort of type II diabetic patients: a global perspective. Kidney Int 2006;69: 2057-2063.

9. United States Renal Data System: 2017 ADR Reference Tables. https://www.usrds.org/reference.aspx (accessed June 19, 2018).

10. Masakane I, Nakai S, Ogata S, et al.: An overview of regular dialysis treatment in Japan (as of 31 December 2013). Ther Apher Dial 2015;19:540-574.

11. Mudaliar S, Polidori D, Zambrowicz B, Henry RR: Sodium-glucose cotransporter inhibitors: effects on renal and intestinal glucose transport: from bench to bedside. Diabetes Care 2015;38:2344-2353.
12. Inagaki $\mathrm{N}$, Kondo $\mathrm{K}$, Yoshinari $\mathrm{T}$, et al.: Efficacy and safety of canagliflozin in Japanese patients with type 2 diabetes: a randomized, double-blind, placebo-controlled, 12-week study. Diabetes Obes Metab 2013;15:1136-1145.

13. Inagaki $\mathrm{N}$, Kondo $\mathrm{K}$, Yoshinari $\mathrm{T}$, et al.: Efficacy and safety of canagliflozin monotherapy in Japanese patients with type 2 diabetes inadequately controlled with diet and exercise: a 24-week, randomized, double-blind, placebocontrolled, phase III study. Expert Opin Pharmacother 2014;15:1501-1515.

14. Polidori D, Mari A, Ferrannini E: Canagliflozin, a sodium glucose co-transporter 2 inhibitor, improves model-based indices of beta cell function in patients with type 2 diabetes. Diabetologia 2014;57:891-901.

15. Inagaki N, Kondo K, Yoshinari T, Kuki H: Efficacy and safety of canagliflozin alone or as add-on to other oral antihyperglycemic drugs in Japanese patients with type 2 diabetes: a 52-week open-label study. J Diabetes Investig 2015;6:210-218.

16. Cherney DZ, Perkins BA, Soleymanlou N, et al.: Sodium glucose cotransport-2 inhibition and intrarenal RAS activity in people with type 1 diabetes. Kidney Int 2014;86:1057-1058.

17. Novikov A, Vallon V: Sodium glucose cotransporter 2 inhibition in the diabetic kidney: an update. Curr Opin Nephrol Hypertens 2016;25:50-58.

18. Vallon V, Thomson SC: Targeting renal glucose reabsorption to treat hyperglycaemia: the pleiotropic effects of SGLT2 inhibition. Diabetologia 2017;60:215-225.

19. Panchapakesan U, Pegg K, Gross S, et al.: Effects of SGLT2 inhibition in human kidney proximal tubular cells-renoprotection in diabetic nephropathy? PLoS One 2013;8:e54442.

20. Heerspink HJ, Desai M, Jardine M, et al.: Canagliflozin slows progression of renal function decline independently of glycemic effects. J Am Soc Nephrol 2017;28:368-375.

21. Neal B, Perkovic V, Mahaffey KW, et al.: Canagliflozin and cardiovascular and renal events in type 2 diabetes. $\mathrm{N}$ Engl J Med 2017;377:644-657.

22. Witte EC, Lambers Heerspink HJ, de Zeeuw D, et al.: First morning voids are more reliable than spot urine samples to assess microalbuminuria. J Am Soc Nephrol 2009;20:436443.

23. Matsuo S, Imai E, Horio M, et al.: Revised equations for estimated GFR from serum creatinine in Japan. Am J Kidney Dis 2009;53:982-992.

24. Horio M, Imai E, Yasuda Y, et al.: GFR estimation using standardized serum cystatin C in Japan. Am J Kidney Dis 2013;61:197-203.

25. de Zeeuw D, Remuzzi G, Parving HH, et al.: Albuminuria, a therapeutic target for cardiovascular protection in type 2 diabetic patients with nephropathy. Circulation 2004;110: 921-927.

26. Japanese Society of Nephrology: Evidence-based clinical practice guideline for CKD 2013. Chapter 9: diabetic nephrology. Clin Exp Nephrol 2014;18:370-374.

27. American Diabetes Association: 3. Comprehensive medical evaluation and assessment of comorbidities: Standards of Medical Care in Diabetes-2018. Diabetes Care 2018;41: S28-S37.

28. Cherney D, Lund SS, Perkins BA, et al.: The effect of sodium glucose cotransporter 2 inhibition with empagliflozin on microalbuminuria and macroalbuminuria in patients with type 2 diabetes. Diabetologia 2016;59:1860-1870. 
29. Heerspink HJ, Johnsson E, Gause-Nilsson I, et al.: Dapagliflozin reduces albuminuria in patients with diabetes and hypertension receiving renin-angiotensin blockers. Diabetes Obes Metab 2016;18:590-597.

30. Petrykiv SI, Laverman GD, de Zeeuw D, Heerspink HJL: The albuminuria-lowering response to dapagliflozin is variable and reproducible among individual patients. Diabetes Obes Metab 2017;19:1363-1370.

31. Howard RL, Buddington B, Alfrey AC: Urinary albumin, transferrin and iron excretion in diabetic patients. Kidney Int 1991;40:923-926.

32. Thomas MC, Burns WC, Cooper ME: Tubular changes in early diabetic nephropathy. Adv Chronic Kidney Dis 2005; 12:177-186.

33. Tang SC, Lai KN: The pathogenic role of the renal proximal tubular cell in diabetic nephropathy. Nephrol Dial Transplant 2012;27:3049-3056.

34. Ferrannini E, Mark M, Mayoux E: CV Protection in the EMPA-REG OUTCOME Trial: a "Thrifty Substrate" Hypothesis. Diabetes Care 2016;39:1108-1114.

35. Sano M, Takei M, Shiraishi Y, Suzuki Y: Increased hematocrit during sodium-glucose cotransporter 2 inhibitor therapy indicates recovery of tubulointerstitial function in diabetic kidneys. J Clin Med Res 2016;8:844-847.

36. Mudaliar S, Alloju S, Henry RR: Can a shift in fuel energetics explain the beneficial cardiorenal outcomes in the EMPA-REG OUTCOME study? A unifying hypothesis. Diabetes Care 2016;39:1115-1122.

37. Cherney DZ, Perkins BA, Soleymanlou N, et al.: Renal hemodynamic effect of sodium-glucose cotransporter 2 inhibition in patients with type 1 diabetes mellitus. Circulation 2014;129:587-597.

38. Lambers Heerspink HJ, de Zeeuw D, Wie L, et al.: Dapagliflozin a glucose-regulating drug with diuretic properties in subjects with type 2 diabetes. Diabetes Obes Metab 2013;15:853-862.

39. Wanner C, Inzucchi SE, Lachin JM, et al.: Empagliflozin and progression of kidney disease in type 2 diabetes. $\mathrm{N}$ Engl J Med 2016;375:323-334.

40. Apperloo AJ, de Zeeuw D, de Jong PE: A short-term antihypertensive treatment-induced fall in glomerular filtration rate predicts long-term stability of renal function. Kidney Int 1997;51:793-797.

41. Holtkamp FA, de Zeeuw D, Thomas MC, et al.: An acute fall in estimated glomerular filtration rate during treatment with losartan predicts a slower decrease in long-term renal function. Kidney Int 2011;80:282-287.

42. Morales E, Millet VG, Rojas-Rivera J, et al.: Renoprotective effects of mineralocorticoid receptor blockers in patients with proteinuric kidney diseases. Nephrol Dial Transplant 2013;28:405-412.

43. Zatz R, Dunn BR, Meyer TW, et al.: Prevention of diabetic glomerulopathy by pharmacological amelioration of glomerular capillary hypertension. J Clin Invest 1986;77: 1925-1930.

44. Heerspink HJ, Perkins BA, Fitchett DH, et al.: Sodium glucose cotransporter 2 inhibitors in the treatment of diabetes mellitus: cardiovascular and kidney effects, potential mechanisms, and clinical applications. Circulation 2016; 134:752-772.

45. Yamagishi S, Matsui T: Advanced glycation end products, oxidative stress and diabetic nephropathy. Oxid Med Cell Longev 2010;3:101-108.

46. Navarro-Gonzalez JF, Mora-Fernandez C, Muros de Fuentes M, Garcia-Perez J: Inflammatory molecules and pathways in the pathogenesis of diabetic nephropathy. Nat Rev Nephrol 2011;7:327-340.

47. Yamout H, Perkovic V, Davies M, et al.: Efficacy and safety of canagliflozin in patients with type 2 diabetes and stage 3 nephropathy. Am J Nephrol 2014;40:64-74.

48. Hahn K, Ejaz AA, Kanbay M, et al.: Acute kidney injury from SGLT2 inhibitors: potential mechanisms. Nat Rev Nephrol 2016;12:711-712.

49. Szalat A, Perlman A, Muszkat M, et al.: Can SGLT2 inhibitors cause acute renal failure? Plausible role for altered glomerular hemodynamics and medullary hypoxia. Drug Saf 2018;41:239-252.

Address correspondence to:

Maki Gouda, BS

Ikuyaku, Integrated Value Development Division Mitsubishi Tanabe Pharma Corporation 17-10 Nihonbashi-Koamicho Chuo-ku

Tokyo 103-8405

Japan

E-mail: gouda.maki@mm.mt-pharma.co.jp 